\title{
DISEÑO DE UN CUESTIONARIO DE DIAGNÓSTICO ACERCA DEL MANEJO DEL CONCEPTO DE VARIABLE EN EL ÁLGEBRA
}

\author{
TRIGUEROS, M. ${ }^{1}$, REYES, A. ${ }^{1}$, URSINI, S. ${ }^{2}$ y QUINTERO, R. ${ }^{2}$ \\ 1 Departamento de Matemáticas. Itam, México. \\ 2 Departamento de Matemática Educativa Cinvestav, México.
}

\begin{abstract}
SUMMARY
This paper reports the first stage of a project investigating college novice students' interpretations and uses of different realisations of the concept of variable, namely: as unknown, as general number, in functional relationships.

In particular, we present and discuss the reliability of a questionnaire that aimed at providing a first students' profile concerning their interpretations and uses of variable. Finally, initial resuits obtained by analysing students' responses are outlined.
\end{abstract}

\section{INTRODUCCIÓN}

Las matemáticas que se requieren en varias carreras universitarias como, por ejemplo, Economía, Administración, Contabilidad y Ciencias Sociales, demandan del estudiante una gran capacidad de abstracción. Los conceptos que las constituyen son complejos y muy estructurados, lo que hace que su comprensión sea imposible si no se sustenta sobre una base muy sólida constituida por las ideas más elementales del álgebra y de la geometría.

El caso del álgebra es particularmente importante. Esta materia es básica para un buen manejo de las nociones de las matemáticas avanzadas, por lo cual se le dedica mucho tiempo de instrucción al nivel de secundaria. Sin embargo, los estudiantes que ingresan a la universidad aún tienen dificultades con la comprensión y manejo de los conceptos elementales. Los resultados de los exámenes de admisión a las universidades y los resultados de las evaluaciones de los alumnos en los cursos propedéuticos de carácter remedial que se imparten en ellas muestran que sus conocimientos de álgebra son pobres.
La identificación de los problemas específicos de los estudiantes en esta materia y de sus causas, así como la búsqueda de formas alternativas de enseñanza diseñadas para superar estos problemas son temas que requieren de investigación. Con el fin de profundizar en la comprensión de las dificultades a las cuales se enfrentan los estudiantes que ingresan a las carreras antes mencionadas para la solución de problemas algebraicos, es conveniente poner atención a un concepto central en esta materia: el concepto de variable. El trabajo que aquí se presenta se origina del interés por comprender mejor cómo los estudiantes interpretan y manejan la variable y de qué manera esto afecta su desempeño en álgebra.

Para iniciar este proyecto se consideró pertinente diseñar un cuestionario que permitiera obtener un perfil inicial de los estudiantes en cuanto a su desempeño con respecto a probiemas que requieren interpretar, manipular y simbolizar la variable en sus diferentes manifestaciones para, posteriormente, llevar a cabo análisis más profundos al respecto. 
En este artículo se presenta el diseño de una primera versión del cuestionario así como el análisis de su fiabilidad en términos de la Teoría clásica de los tests. Es conveniente señalar que un acercamiento de este tipo no es común entre los investigadores en enseñanza de las matemáticas, dado que se suele considerar el empleo de métodos cualitativos más apropiado para realizar investigaciones en esta disciplina (Freudenthal, 1982; Puig, 1996); sin embargo, si bien es cierto que, para profundizar en la comprensión de las concepciones de los estudiantes, nuestro proyecto global incluye un acercamiento de tipo cualitativo, consideramos que un cuestionario que satisfaga algún criterio de fiabilidad puede proporcionar los primeros elementos para orientar un estudio en profundidad.

\section{ACERCA DEL CONCEPTO DE VARIABLE}

El concepto de variable es de fundamental importancia en el desarrollo y comprensión de cualquier rama de las matemáticas. Este concepto aparece por primera vez en la enseñanza dentro del marco de los cursos de álgebra, aunque no se introduce explícitamente. En dichos cursos, los estudiantes se enfrentan a los procesos de generalización y de modelación que han guiado el desarrollo de las matemáticas, dejando atrás el manejo de operaciones que se aplican exclusivamente a números específicos. Estos procesos requieren de una buena comprensión del concepto de variable, así como de sł adecuado manejo en situaciones diversas.

Fi concepto de variable es complejo. Cuando se revisa su papel dentro del álgebra se encuentra que este concepto se usa con significados diversos en diferentes contextos y que dependiendo de ellos se maneja de distintas maneras. Esta variedad en las formas de empleo hace que el concepto de variable sea difícil de definir y puede ser la causa de muchas de las dificultades que suelen enfrentar los estudiantes (Wagner, 1981, 1983; Usiskin, 1988).

Un usuario competente del álgebra puede asignarle diferentes significados a la variable dependiendo del problema que se le presenta: distingue ecuaciones de tautologías, puede simplificar expresiones y manejar la idea de variación en relaciones funcionales. Estas manifestaciones diversas, que para un experto no resultan problemáticas, pueden convertirse para un novato en un obstáculo dificil de salvar, como lo muestran los estudios que realizó Matz (1982) con estudiantes que se iniciaban en el estudio del álgebra.

Aquí consideraremos tres formas distintas en las que la variable suele usarse en el álgebra escolar y que caracterizaremos de la siguiente manera: la variable como incógnita, cuyo valor se puede determinar con exactitud tomando en consideración las restricciones del problena; la variable como número general, es decir, aquélla que aparece en generalizaciones y en métodos generales; y la variable en una relación de variación conjunta con otras variables que denominaremos variable en relación funcional.
Diversas investigaciones reportan que tanto los estudiantes que se inician en el estudio del álgebra como los más avanzados tienen dificultades con cada uno de estos usos de la variable (más detalles en Ursini, 1994a). listas investigaciones se han restringido por lo general a estudiar el desempeño de alumnos de entre 12 y 16 años, edades en las que se estudia el álgebra en la escuela. Casi no se ha hecho investigación con estudiantes de mayor edad. No se tiene, por lo tanto, información que indique si los estudiantes de niveles superiores han superado estas dificultades o si ellas persisten y eventualmente afectan su descmpeño en matemáticas más avanzadas.

Los estudiantes que inician sus estudios universitarios han cursado al menos cinco años de matemáticas en la escuela secundaria y preparatoria en los cuales, si bien se abordan diversos temas, el énfasis se pone en el manejo del álgebra. A pesar de esto los profesores universitarios suelen quejarse de que los estudiantes continúan mostrando deficiencias en el manejo de expresiones algebraicas. Esto repercute negativamente en sus estudios del cálculo diferencial e integral, así como en el de otras materias de matemáticas avanzadas o en aquéllas que usan las matemáticas como herramienta.

Es posible que, en buena medida esas dificultades provengan de la falta de una construcción adecuada, por parte de los estudiantes, del concepto de variable. Esta construcción debería incluir todos los aspectos mencionados anteriormente y la posibilidad de pasar de uno a otro con flexibilidad y acoplándose a las exigencias de los problemas que se intentan resolver.

Si se parte de la hipótesis de que el manejo de la variable requiere de la capacidad de diferenciar entre sus distintas caracterizaciones, un paso importante en la comprensión de la forma en la que este concepto se aprende es la inđagación acerca de cómo los estudiantes usan e interpretan cada una de ellas. Un diagnóstico adecuado permitiría intervenir mediante un rediseño de la didáctica de este concepto que tome como punto de partida las concepciones de los estudiantes y las dificultades que ahí se originan.

\section{EL INSTRUMENTO DIAGNÓSTICO}

EI cuestionario que diseñamos consiste de 52 preguntas cuya solución no requiere de un manejo algebraico complejo y en las que se trata de poner énfasis, separadamente, en cada una de las caracterizaciones de la variable. Este diseño pretende facilitar un diagnóstico acerca de cómo los alumnos interpretan, manipulan y simbolizan cada una de las distintas manifestaciones de Ia variable y con cuáles tienen más dificultades.

A continuación se especifican las capacidades que se requieren para manejar las caracterizaciones de la variable antes mencionadas. Dichas capacidades, usadas como indicadores, permiten diseñar ítems precisos, para medirlas usando métodos cuantitativos. 
- La variable como incógnita. Un manejo adecuado de la variable como incógnita implica la capacidad de:

- interpretar la variable como un número cuyo valor o valores específicos se pueden determinar a partir de las restricciones de un problema dado;

- manipular los elementos que componen una ecuación, parámetros e incógniła, independientemente de la operación o las operaciones involucradas;

- identificar y simbolizar la incógnita en problemas específicos, susceptibles a representarse mediante una ecuación.

- La variable como un número general. Lo que se espera aquí del estudiante es la capacidad de:

- interpretar la variable como representación de un número cualquiera en expresiones algebraicas tales como tautologías y expresiones abiertas;

- manipular la variable en ese tipo de expresiones;

- identificar y simbolizar el objeto general en situaciones particulares que pueden describirse en términos de una regla o método general.

- La variable en una relación funcional. La idea de relación funcional puede considerarse desde dos perspectivas: una, estática, en la que la relación se concibe como la correspondencia punto por punto entre dos conjuntos de valores; otra dinámica, en la que se resalta la variación interdependiente de las variables. El manejo competente de este uso de la variable implica la capacidad de:

- reconocer las relaciones funcionales (en su representación analítica, gráfica o tabular) e interpretar las variables involucradas en forma tanto estática cuanto dinámica, dependiendo de la naturaleza del problema;

- manipular las variables para determinar los valores o intervalos de variación que cada una de ellas puede tomar en términos de la otra;

- simbolizar situaciones que involucran una relación funcional.

Estos tres usos del concepto de variable están fuertemente interrelacionados. En la mayoría de los problemas a los que se enfrenta un estudiante en la escuela aparecen conjuntamente, aunque cada una puede sobresalir en distintas etapas de la resolución del problema. Para ilustrar lo anterior recurrimos al ya conocido ejemplo de Usiskin (1988):

\section{Encuentra la ecuación de la línea que pasa por el punto $(6,2)$ y cuya pendiente es 11 .}

(Usiskin, 1988, p. 14)

Cuando, para resolver este problema, se parte de la relación general que existe entre los puntos de una línea y su pendiente, a saber: $Y=m X+b$, queda implícito que se espera que el estudiante sea capaz de concebir las variables como números generales. En efecto, esta expresión describe una línea general y las variables invoIucradas representan números generales que pueden, por Io tanto, asumir cualquier valor. Sin embargo, para una línea particular, $m$ y $b$ no representan números generales, sino constantes. Por ejemplo, en el ejemplo arriba mencionado el valor de la pendiente está dado y tiene que substituirse por m. $b$ es una incógnita que puede determinarse usando los datos. $X$ e $Y$ son dos variables vinculadas por una relación funcional: $X$ puede considerarse un argumento al que se le puede asignar cualquier valor mientras que los valores de $Y$ cambian en correspondencia.

Es decir, para resolver este problema, los estudiantes deben ser capaces de trabajar con números generales, con constantes, con incógnitas, con variables en una relación funcional y poder pasar de una a otra interpretación, aun cuando estas diferentes caracterizaciones de la variable tengan la misma representación simbólica.

Sin embargo, al diseñar el instrumento que aquí se discute se buscaron ftems que permitieran diferenciarlas Io más claramente posible; es decir, se buscaron preguntas en las que el énfasis en uno de los aspectos pudiera percibirse con claridad.

\section{CATEGORÍAS DE ANÁLISIS}

Para cada una de las tres caracterizaciones de la variable consideradas se incluyeron ítems que permitían observar si los estudiantes:

- interpretaban correctamente la variable involucrada; - tenían la capacidad para simbolizar una situación en la que aparecía cierta caracterización de la variable;

- eran capaces de manipular las variables que aparecían en una expresión.

Adicionalmente, para tener más información acerca de la comprensión de las variables que aparecen en una relación funcional, se incluyeron ítems que requerían de graficación. La posibilidad de representar globalmente la información que proporciona una fórmula o una tabla mediante una gráfica es una importante manifestación de la comprensión integrai de este aspecto de la variable.

En la siguiente tabla se presenta el númcro de ftems que se dedicaron a explorar cada uno de estos aspectos.

\begin{tabular}{|l|c|c|c|}
\hline & Incógnita & $\begin{array}{c}\text { Número } \\
\text { generalizado }\end{array}$ & $\begin{array}{c}\text { Relación } \\
\text { funcional }\end{array}$ \\
\hline Interpretación & 5 & 4 & 11 \\
\hline Simbolización & 2 & 7 & 5 \\
\hline Manipulación & 1 & 7 & 8 \\
\hline Graficación & - & - & 2 \\
\hline Total de f́tems & 8 & 18 & 26 \\
\hline
\end{tabular}


Como se puede observar en la tabla, el instrumento no contempla el mismo número de ítems para cada una de las distintas caracterizaciones de la variable. El número dedicado a explorar la comprensión de la variable como incógnita es notablemente menor. Esto se debe a que se consideró que en la escuela se suele hacer un énfasis muy grande en este uso de la variable, por io que es de esperar que sea ésta la caracterización de la variable que mejor manejen los estudiantes que inician los estudios universitarios. Por esta razón, se deciđió abordar preferencialmente los otros dos aspectos del concepto sin descuidar, sin embargo, el aspecto de la variable como incógnita.

\section{ACERCA DE LAS FUENTES}

El cuestionario consta de 52 preguntas. De éstas, 35 se tomaron del cuestionario de Ursini (1994b) diseñado para aplicarse a niños de 12-13 años de edad que empezaban el ciclo de educación secundaria y conformado, a su vez, por algunos ítems tomados del proyecto Concepts in Secondary Mathematics and Science (CSMS) (Hart et al., 1981), del proyecto de preálgebra DIME (Geoff Giles), de Rojano (1985) y de Mason (1985). A pesar de la diferencia de edades entre esos niños y los estudiantes a los que iba dirigido nuestro instrumento, se consideró oportuno usar las mismas preguntas dado que su sencillez permite observar claramente la capacidad del estudiante para interpretar, simbolizar y manipular los distintos usos de la variable. Sin embargo, a esas 35 preguntas se añadicron otras 17 elaboradas expresamente para este cuestionario. En éstas se abordan problemas de graficación y de simbolización de relaciones funcionales, así como de manejo de tablas numéricas.

L.os ítems son de respuesta abierta, lo que permite, por una parte, hacer un análisis cuantitativo, utilizando la teoría clásica de los tests; y por otra, llevar a cabo un análisis mediante métodos de investigación cualitativa con el fin de adentrarse un poco más en las dificultades específicas de los estudiantes. Este análisis complementado con entrevistas centradas en puntos detectados en esta primera etapa forma parte de nuestro proyecto de investigación y se realizará próximamente.

\section{PILOTEO DEL CUESTIONARIO}

El instrumento se probó con una muestra de 73 estudiantes escogidos entre los 450 que fueron aceptados para iniciar sus estudios universitarios en el Instituto Tecnológico Autónomo de México (ITAM). Si bien ésta es una institución privada, cl conjunto de estudiantes que ingresa a ella tiene conocimientos semejantes al conjunto general de los estudiantes que han terminado la preparatoria y, con ello, sus estudios de álgebra. La muestra escogida se puede, por lo tanto, considerar representativa de los alumnos que empiezan sus estudios universitarios.

La elección de la muestra se hizo tomando entre los estudiantes de primer ingreso al ITAM, a los matriculados en el primer curso de matemáticas que se imparte en verano. A to largo de los años se ha observado que las calificaciones de este curso en período de verano se distribuyen de la misma manera que las de otros períodos del año, por lo tanto, se puede considerar que la selección de lá muestra no fue sesgada.

\section{ANÁLISIS CUANTITATIVO DEL CUESTIONARIO PILOTO}

\section{Medidas de dispersión y fiabilidad del cuestionario}

El cuestionario piloto se analizó utilizando la teoría clásica de los tests (Muñiz, 1992). Se calculó el coeficiente de fiabilidad del cuestionario completo así como la fiabilidad de cada una de las partes que lo componen. A saber, el conjunto de preguntas que exploran el manejo de la variable como número generalizado es el primer subtest; las que exploran la variable como incógnita forman el segundo subtest, y el tercero está formado por las preguntas que exploran la variable en una relación funcional. Para calcular la frabilidad se utilizó el coeficiente Spearman-Brown que se calcula con las varianzas de cada una de las preguntas del test y las correlaciones entre ellas.

A partir de la matriz de las respuestas de los estudiantes se hizo el diagrama de frecuencias de acierto para cada una de las preguntas, que se muestra en la figura que se anexa. El número de reactivos en el cuestionario fue de 52 ; no hubo estudiantes con una puntuación inferior a 17 aciertos ni superior a 48 . La moda ocurrió en la puntuación 41 con 8 alumnos, quedando en segundo lugar la puntuación 34 , con 7 . Se realizaron algunas estimaciones que se incluyen en el gráfico de la página siguiente.

El resultado de la puntuación media es más bajo del esperado para alumnos de nivel universitario. Esto puede considerarse como un indicador de que el concepto de variable no se ha aprendido sólidamente en la escuela. La varianza es grande y esto indica una dispersión de la puntuación obtenida mayor que la esperada para alumnos que han cursado álgebra en varias ocasiones. Las desviaciones estándar de los errores están dentro de los márgenes usuales.

Utilizando la fórmula de Spearman-Brown para el cuestionario completo, se obtuvo el valor de:

$$
\rho^{*}{ }_{x x}=0.880886
$$

que indica un buen nivel de fiabilidad.

El mismo análisis se realizó para cada uno de los $s u b$ tests. Esta información se resume en el siguiente cuadro:

\begin{tabular}{|c|c|c|ccc|}
\hline Tema & $\begin{array}{c}\text { Núm. de } \\
\text { preguntas }\end{array}$ & $\begin{array}{c}\text { Media } \\
\text { muestral }\end{array}$ & $\begin{array}{c}\text { Varianza } \\
\text { estimada }\end{array}$ & $\begin{array}{c}\text { Coef. } \\
\mathrm{S}-\mathrm{B}\end{array}$ \\
\hline Núm. generajizado & 18 & 11,19 & 11,18 & 0,81 \\
\hline Incógnita & 8 & 7,44 & 0,44 & 0,06 \\
\hdashline Variabie & 26 & 17 & 17,5 & 0,8 \\
\hline
\end{tabular}


Frecuencias de alumnos por aciertos

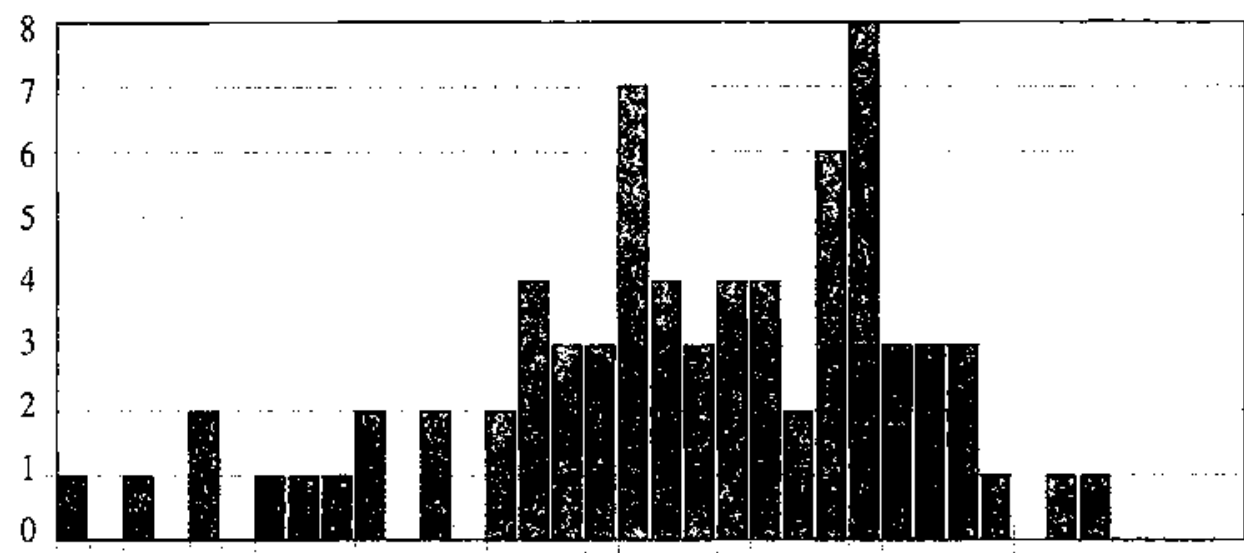

$\begin{array}{llllllllllllllllll}17 & 19 & 21 & 23 & 25 & 27 & 29 & 31 & 33 & 35 & 37 & 39 & 41 & 43 & 45 & 47 & 49 & 51\end{array}$

Aciertos

$\left[\begin{array}{ll}\text { Puntuación media } & \mathrm{X}=35,63 \\ \text { Varianza de la puntuación } & \mathrm{S}_{\mathrm{x}}^{2}=45,7363 \\ \text { Desviación estándar del error } & \mathrm{S}_{\mathrm{c}}=3,120252 \\ \text { Desviación estándar de los errores de estimación } & \mathrm{S}_{\mathrm{vx}}=2,768295\end{array}\right.$

La fiabilidad de los subtests es bastante buena excepto para aquél referido a la variable como incógnita. Esto puede deberse a la longitud tan reducida del mismo. Asimismo es interesante notar que la varianza estimada es muy pequeña, lo cual está reflejando mucha similitud entre las puntuaciones obtenidas.

Al diseñar el cuestionario se decidió incluir menos ítems relativos a este uso de la variable por ser éste el aspecto más enfatizado en la docencia y se seleccionaron ítems de dificultad similar a la de los demás. Los resultados que aquí mostramos indican que este subtest es poco confiable y sería conveniente reemplazarlo por otro que contenga un mayor número de preguntas y de mayor dificultad para que se cubran más cabalmente las distintas posibilidades de uso de la incógnita.

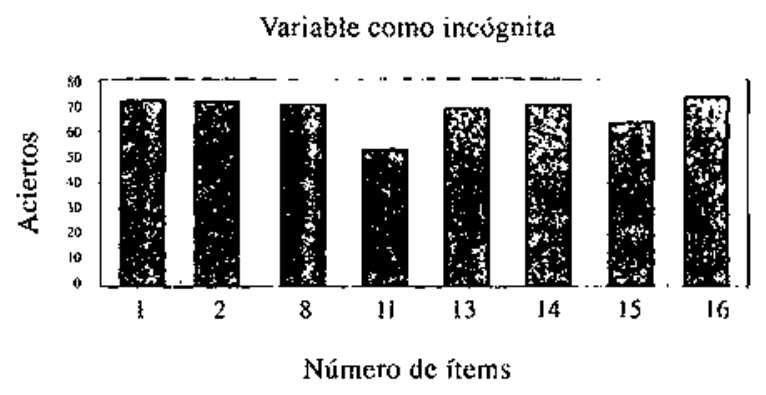

Al estudiar el comportamiento de los otros subtests, vemos que la variabilidad en las calificaciones obtenidas es mucho mayor que en el anterior y que los índices de fiabilidad son también mucho mayores.

Variable como número generalizado

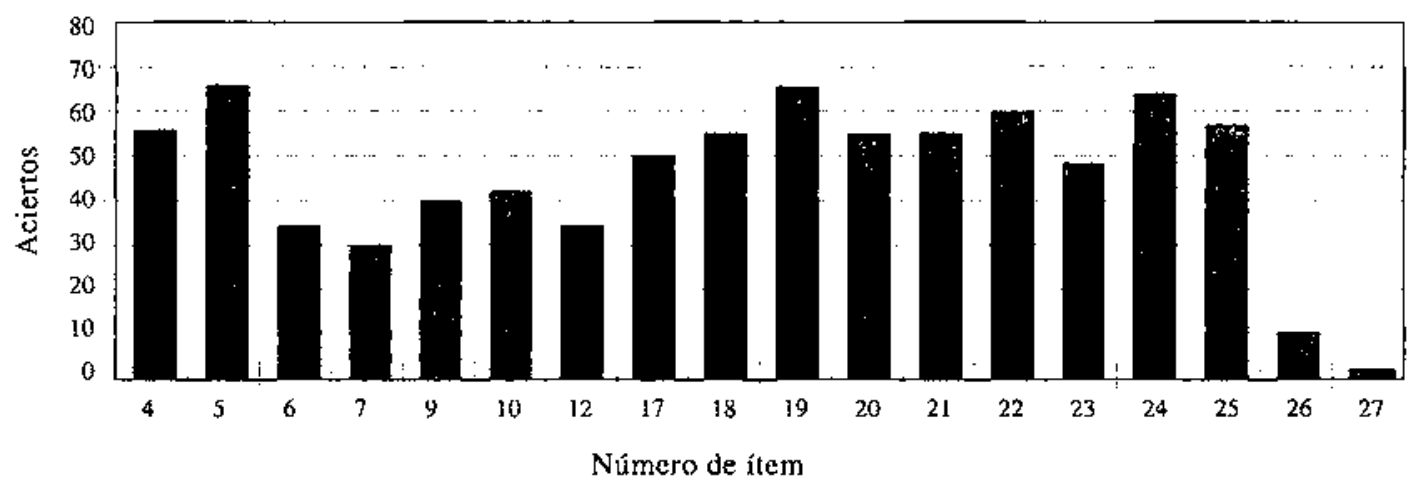


Variable en la función

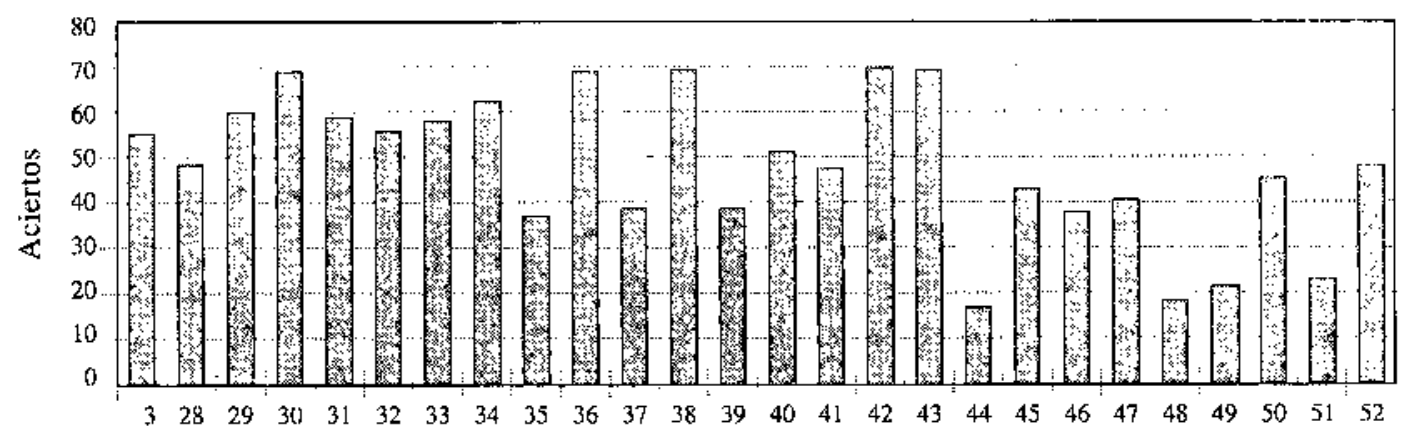

Número de ítem

I a varianza en cstos subtests nos muestra mucha variabilidad en las respuestas de los estudiantes. Esto es un indicador de la falta de solidez en el manejo del concepto. aciertos es menor que la que podría esperarse dada la escolaridad de los estudiantes y que ćsta baja aún más

\begin{tabular}{|c|c|c|c|c|c|c|c|c|c|c|c|c|c|c|c|c|c|c|c|}
\hline Núm. preg. & 1 & 2 & 3 & 4 & 5 & 6 & 7 & & 8 & 9 & & 10 & 11 & 12 & 13 & 14 & 15 & 16 & 17 \\
\hline Aciertos & 73 & 72 & 59 & 57 & 65 & 38 & 31 & & 70 & 42 & & 41 & 53 & 34 & 69 & 69 & 65 & 73 & 49 \\
\hline Frrores & 0 & 1 & 14 & 12 & 4 & 35 & 40 & & 3 & 29 & & 30 & 17 & 37 & 4 & 4 & 8 & 0 & 23 \\
\hline $\begin{array}{l}\text { No } \\
\text { tespondidas }\end{array}$ & 0 & 0 & 0 & 4 & 4 & 0 & 2 & & 0 & 2 & & 2 & 3 & 2 & 0 & 0 & 0 & 0 & 1 \\
\hline Nún. preg. & 18 & 19 & 20 & 21 & 22 & 23 & 24 & & 25 & 26 & & 27 & 28 & 29 & 30 & 31 & 32 & 3.3 & 34 \\
\hline Aciertos & 58 & 64 & 53 & 53 & 60 & 47 & 63 & & 57 & 5 & 9 & 1 & 49 & 48 & 60 & 48 & 68 & 57. & 55 \\
\hline Errores & 14 & 9 & 18 & 19 & 12 & 23 & 6 & & 15 & 4 & & 29 & 20 & -19 & 9 & 23 & 5 & 11 & 14 \\
\hline $\begin{array}{l}\text { No } \\
\text { respondidas }\end{array}$ & I & 0 & 2 & 1 & 1 & 3 & 4 & & 1 & 23 & & 43 & 4 & 6 & 4 & 2 & 0 & 5 & 4 \\
\hline Núm. preg. & 35 & 36 & 37 & 38 & 39 & 40 & 41 & 42 & 4 & 3 & 44 & 45 & 46 & 47 & 48 & 49 & 50 & 51 & 52 \\
\hline Aciertss & 67 & 64 & 45 & 69 & 41 & 51 & 46 & 70 & 6 & 9 & 18 & 43 & 38 & 40 & 19 & 22 & 46 & 21 & 49 \\
\hline Lirrores & 2 & 6 & 9 & 1 & 20 & 14 & 19 & 1 & & 1 & 53 & 25 & 31. & 28 & 40 & 12 & 19 & 45 & 16 \\
\hline $\begin{array}{l}\text { No } \\
\text { respondidas }\end{array}$ & 4 & 3 & 19 & 3 & 12 & 8 & 8 & 2 & & 3 & 2 & 5 & 4 & 5 & 14 & 39 & 8 & 7 & 8 \\
\hline
\end{tabular}

\section{Análisis de las preguntas}

En el siguiente cuadro se muestra la frecuencia de aciertos en las respuestas dadas a cada una de las preguntas.

Fin este cuadro se puede apreciar que la frecuencia de cuando las preguntas involucran la variable como número general o en relación funcional.

Se hizo además el análisis para medir el índice de dificultad (ID) de cada una de las preguntas en rejación a las otras. La forma de calcular el índice de dificultad consiste en dividir el total de personas que acertaron por la población; por lo tanto, un índice de dificultad 1 indica

\begin{tabular}{|c|c|c|c|c|c|c|c|c|c|c|c|c|c|}
\hline İtem & 1 & 2 & 3 & 4 & 5 & 6 & 7 & 8 & 9 & 10 & 11 & 12 & 13 \\
\hline ID & 1 & 986 & .795 & 826 &, 942 & ,493 & .437 & 959 &, 563 & 577 &, 757 & 479 &, 945 \\
\hline
\end{tabular}

\begin{tabular}{|l|c|c|c|c|c|c|c|c|c|c|c|c|c|}
\hline Ittem & 14 & 15 & 16 & 17 & 18 & 19 & 20 & 21 & 22 & 23 & 24 & 25 & 26 \\
\hline ID &, 945 &, 877 & 1 &, 681 &, 792 &, 877 &, 746 &, 736 &, 833 &, 671 &, 913 &, 792 &, 180 \\
\hline
\end{tabular}

\begin{tabular}{|l|c|c|c|c|c|c|c|c|c|c|c|c|c|}
\hline Ítem & 27 & 28 & 29 & 30 & 31 & 32 & 33 & 34 & 35 & 36 & 37 & 38 & 39 \\
\hline ID & 033 &, 710 &, 507 &, 870 &, 676 &, 932 &, 838 &, 797 &, 928 &, 914 &, 833 &, 986 &, 656 \\
\hline
\end{tabular}

\begin{tabular}{|l|c|c|c|c|c|c|c|c|c|c|c|c|c|}
\hline Ítern & 40 & 41 & 42 & 43 & 44 & 45 & 46 & 47 & 48 & 49 & 50 & 51 & 52 \\
\hline ID &, 785 &, 708 &, 986 &, 986 &, 254 &, 618 &, 536 &, 588 &, 322 &, 647 &, 708 &, 318 &, 754 \\
\hline
\end{tabular}


que todos la contestaron correctamente y que la pregunta es muy fácil. Los resultados se muestran en el siguiente cuadro:

El análisis de las preguntas muestra lo siguiente:

- Las preguntas 1 y 16 (sombreadas) fueron contestadas correctamente por el $100 \%$ de los alumnos. Ambas preguntas se refieren a la variable como incógnita: su simbolización y su interpretación, respectivamente.

- Las preguntas 2, 8, 42 y 43 (sombreadas) fueron contestadas correctamente por más del $95 \%$ de los alumnos que lo intentaron. Dos de estas preguntas se refieren nuevamente al concepto de variable como incógnita, las otras dos tienen que ver con la manipulación de la variable en la relación funcional.

- Sólo para 8 reactivos $(6,7,12,26,27,44,48$ y 51$)$, menos de la mitad de alumnos contestaron acertadamente. Las cuatro primeras de estas preguntas se refieren a la variable como número generalizado y las tres últimas a Ia variable como función.

- Las preguntas de mayor grado de dificultad fueron la 26 , Ia 27 y la 44 (celdas con doble marco). En las dos primeras se trata de la simbolización de un número generalizado. La tercera se refiere a la manipulación de las variables en la relación funcional y resultó ser la menos difícil de las tres. La pregunta 27 resultó ser la más difícil de todas, debido quizá a que involucra la idea de cambio.

Por otro lado, se calculó el índice de discriminación de cada una de las preguntas y se obtuvieron las siguientes conclusiones:

- Los reactivos con mayor poder discriminatorio fueron el 25 y el 45 seguidos por el $12,18,22,23,34,35$ y 44 .

Ias preguntas $1,6,13,16$ y 48 tienen correlaciones extremadamente bajas, lo que indica su poco poder para discriminar, por lo que habría que modificarlas.

En lo generai se encontró que únicamente el $27 \%$ del total de preguntas fueron acertadas por más del $95 \%$ de los estudiantes. Este resultado es sorprendente en estudiantes de nivel universitario y comparado con los resultados de las investigaciones señaladas al inicio del trabajo y realizadas con estudiantes que inician el estudio del álgebra. Parece indicar que la enseñanza escolar no ayuda a los estudiantes a superar las dificultades que encuentran en el manejo de la variable en sus distintas manifestaciones.

\section{CONCLUSIONES}

El análisis del cuestionario propuesto nos permite obtener las siguientes conclusiones:

En términos generales, el cuestionario es confiable y consistente. Permite adentrarse en las concepciones que los estudiantes tienen acerca de los diferentes usos de la variable.

La varianza en la respuesta a las preguntas concernientes a la variable como número generalizado y a la variable en la relación funcional fue grande y hubo variación en sus índices de dificultad.

Las preguntas referentes a la variable como incógnita resuitaron las de mayor frecuencia de respuesta y menor varianza al analizarse mediante la teoría clásica de los tests. Analizadas como subtest, tienen un índice bajo de fiabilidad, por lo que serf́a conveniente modificar esta parte en una nueva versión del instrumento.

Las puntuaciones obtenidas por los estudiantes fueron notablemente bajas para las preguntas que involucran la variable como número general. Dada una generalización, no todos los sujetos pudieron simbolizaria (preguntas $3,4,5,6$ ). Tampoco lo pudieron hacer cuando para ello tenían que usar un símbolo ya dado (preguntas $17,18,19,20,21,22,23,24,25)$. La gran mayoría mostró dificultades para generalizar a partir de un patrón geométrico (preguntas 26, 27). Esto indica fuertes dificultades con la generalización, su interpretación y expresión.

La gran variabilidad en las respuestas a las preguntas que involucraban variables en relación funcional sugiere un pobre dominio del concepto. Las respuestas dadas a las preguntas que requerían la determinación de intervalos numéricos (preguntas 44,45 ) sugieren que los estudiantes enfrentan serias dificultades para trabajar con esta noción; se observó una tendencia a describir los intervalos en términos de números naturales o enteros. Las respuestas de los estudiantes muestran su incapacidad para concebir la variación de manera dinámica, lo cual puede ser el resultado de un énfasis exagerado en la instrucción en el aspecto estático de la función, esto es, en la correspondencia entre variables.

Estos resuItados sugieren que los estudiantes universitarios tienen una concepción muy pobre e inestable de la variable y ponen también en evidencia algunos puntos en los que habrá que profundizar en las etapas sucesivas de la investigación.

A partir del análisis del cuestionario se tomaron decisiones respecto al instrumento mismo, con el objeto de adecuarlo a los objetivos de la investigación. Entre estas decisiones destacan las siguientes:

A fin de lograr una mayor fiabilidad del subtest correspondiente a la variable como incógnita, se decidió añadir algunas preguntas relativas a su manipulación y a su simbolización y quitar aquéllas que fueron respondidas correctamente por todos los estudiantes. Se decidió que los nuevos f́tems deberfan tener un grado de dificultad ligeramente mayor con el objeto de obtener información más clara a partir de las respuestas de los estudiantes.

Se eliminó el ítem 19, único f́fem en el que aparece una desigualdad, por no proporcionar información suficiente y se sustituyó por otro que involucra una tautología de 
Se cambió la redacción de algunos ftems con el fin de esclarecer si las dificultades presentadas por los estudiantes se deben a este factor o a la falta de comprension del concepto que nos interesa.

Aquellos ítems en los que se pedía una lectura directa de una tabla o de una gráfica, una interpolación o la identificación del papel de las variables, como el 40, 41 y 50, se sustifuyeron por otros por considerarse que no aportaban sufficiente información sobre el aspecto de Ia variable al que se habían relacionado al hacer el diseño del instrumento.

Los ítems 48 y 49 se eliminaron también y se sustituyeron. En este caso porque, al no ser posibie encontrar una relación sencilla entre las variables involucradas en el problema, no era fácil interpretar las respuestas proporcionadas por los estudiantes.

El nuevo cuestionario volverá a aplicarse a una muestra de estudiantes semejante a la que se utilizó en el caso del cuestionario que aquí se reporta. Las respuestas de los estudiantes se analizarán cuantitativa y cualitativamente y se clasificarán con el fin de interpretar sus concepciones acerca del concepto de variable y para establecer una base sobre la cual se llevarán a cabo entrevistas que permitirán profundizar en la comprensión de la forma en

\section{REFERENCIAS BIBLIOGRÁFICAS}

FREUDENTHAI, H. (1982). Fiabilité, validité et pertinence critères de la recherche sur l'enseignement de la mathématique. Educational Studies in Mathematics, 13, pp. 395-408.

GILES GEOFF, DIME Pre-Algebra Project, Number Pattern 1Simple Mapping, Department of Education, University of Stirling.

HARTK, M., KERSLAKF, D., BROWN, M.L., RUDDOCK, G., KUCHFMANN, D.E. y MCCARTNEY, M. (1981). Children's Understanding of Mathematics, I I-16, John Murray.

MASON, J., GRAHAM, A., PIMM, D. y GOWAR, N. (1985). Routes to Roots of Algebra. Inglaterra: The Open University Press.

MA'T7, M. (1982), Towards a Process Model for High School Algebra Errors, en Sleeman, D. y Brown, J.S. (eds.). Intelligent Tutoring Systems. Londres: Academic Press.

MUÑI7, I. (1992). Teoría clásica de los tests. España: Ediciones Pirámide

PUIG, L. (1996). La didáctica de las matemáticas como tarea investigadora, en Puig, L. y Calderón, J. (eds.). Investigación la que estos estudiantes entienden el concepto de variable.

El diseño y análisis del cuestionario constituye la primera etapa del estudio. Posteriormente se hará un análisis cualitativo de las repuestas de los alumnos, lo que permitirá detectar sus dificultades y deficiencias. Esta primera fase nos indicó ya algunos puntos en los que hay que enfocar la atención en el análisis cualitativo de las respuestas, que se presentará posteriormente:

Las respuestas de los estudiantes indican que su concepción de la variable como número generalizado y en la relación funcional es pobre. Los alumnos tienen dificultad para concebir la variación en una forma dinámica

No obstante que el cuestionario se diseñó con la idea de explorar los conocimientos más elementales respecto a la variable y se aplicó a estudiantes que han cursado al menos cuatro años de esta materia, las puntuaciones obtentdas por los estudiantes fueron bajas. La media de 35,63 sobre 52 preguntas es alarmantemente baja.

Las dificultades de los estudiantes se agudizan cuando los ítems no se resuelven mediante manipulación. I a interpretación de la variable les causa problemas y su simbolización aún más. y didáctica de las matemáticas, pp. 103-107. Madrid: CIDF/ MEC.

ROJANO, T. (1985). De la aritméticá al álgebra (un estudio clínico con niños de 12 a 13 años de edad). 'Tusis doctoral. Sección de Matemática Educativa. México: CINVESTAV.

URSINI, S. (1994a). Los niños y las variables. Educación Matemática, 6(3). Grupo Editorial Iberoamérica.

URSINI, S. (1994b). Pupils' Approaches to Different Characterizations of Variable in Logo. PhD Thesis. University of London Institute of Education.

USISKIN, Z. (1988). Conceptions of School AIgebra and Uses of Variables, en Coxford A.F. y Shulte A.P. (eds.). The Ideas of Algebra $K-12$, pp. 8-19.

WAGNER, S. (198I). An Analytical Framework for Mathematical Variables. Proceedings of the Fifth PME Conference, pp. 165-170. Grenoble, Francia.

WAGNER, S. (1983). What are These Things Called Variables? Mathematics Teacher, October, 1983, pp. 474-479. 


\section{ANEXO 1}

\section{CUESTIONARIO}

I. En este ejercicio, solamente escribe una fórmula. NO CALCULES el número.

1. Fscribe una fórmula que exprese: Un número desconocido sumado a 5 es igual a 8 .

2. Fscribe una fórmula que exprese: Un número desconocido multiplicado por 13 es jgual a 127.

3. Hscribe una fórmula que exprese: Un número desconocido es igual a 6 más otro número desconocido.

4. Escribe una fórmula que exprese: multiplica a 8 por la suma de 3 y un número desconocido.

5. Escribe una fórmula que exprese: 4 sumado a $n+5$.

6. Escribe una formula que exprese: un número desconocido dividido por 5 y el resultado sumado a 7.

II. Para cada una de las siguientes expresiones escribe los valores que piensas que puede tener la letra. Si piensas que hay más de uno, escribe algunos de ellos.

7. $x+2=2+x$

8. $3+y=7$

9. $x=x$

10. $4+s$

11. $x+5=x+x$

12. $3+a+a+a+10$

III. Para cada una de las siguientes expresiones escribe los valores que piensas que puede tomar Ia literal:

13. $z+874=1093$

14. $525+x=823$

15. $4+x=2$

16. $3 \times \mathrm{m}=15$

IV. El perímetro de una figura se calcula sumando la longitud de sus lados. Escribe la fórmula que expresa el perímetro de cada una de las siguientes figuras.

17

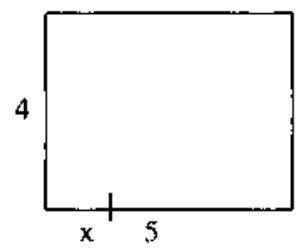

18.

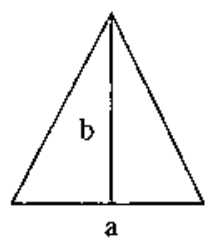


19. Escribe la fómula que expresa «Un número desconocido es mayor que 5 y traza sobre la recta real los números a los que corresponde.

V. Escribe una fórmula para calcular el área de las siguientes figuras:

20

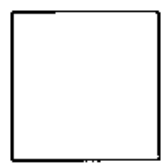

$\mathrm{X}$

23.

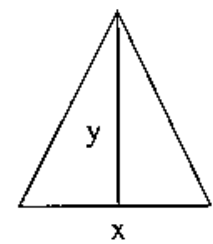

21.

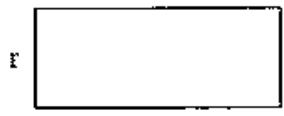

s

24. En la siguiente figura, el polígono no es completamente visible. Debido a que no sabemos cuántos lados tiene en total, diremos que tiene N lados. Cada lado mide 2 centímetros de longitud. Escribe una fórmula que calcule el perímetro de la figura.

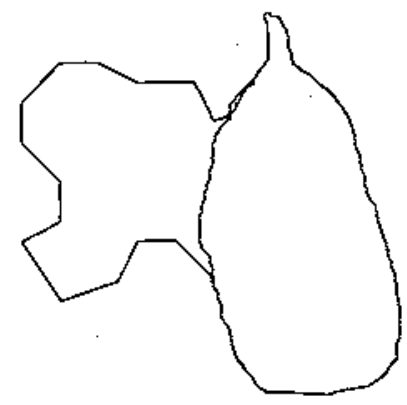

\section{Observa las siguientes figuras:}

Número de puntos

Figura \# 1

Figura \#2

Figura \# 3

$$
\begin{aligned}
& 000 \\
& 000 \\
& 000
\end{aligned}
$$

Figura \#3

$$
\begin{aligned}
& 00009 \\
& 0000 \\
& 0000 \\
& 0000
\end{aligned}
$$


¿Cuántos puntos hay en la figura \#4?

Dibuja una figura semejante para el número 5 y da el número total de puntos.

Dibuja una figura semejante para el número 6 y da el número total de puntos.

25. Imagínate que puedes seguir dibujando figuras hasta la figura m. ¿Cuántos puntos en totał tendrá la figura m?

\section{Si para hacer las figuras del ejercicio anterior vas agregando puntos}

¿Cuántos puntos agregas para pasar de la fígura I a la 2 ?

¿Cuántos puntos agregas para pasar de la figura 2 a la 3 ?

26. Escribe una fórmula que exprese cómo vas agregando puntos a la figura 1 hasta llegar a la figura $m$.

27. Escribe una fómula que relacione cómo vas agregando puntos hasta llegar a la fígura $m$ con el total de puntos que tiene la figura $m$.

28. Si $x+3=y$ ¿qué valores puede tomar $x$ ?

29. ¿Qué valores puede tomar $y$ ?

30. ¿Existe alguna relación entre los valores de $x$ y $y$ ? Subraya la respuesta correcta.

$$
\text { Sí No No sé }
$$

31. Si $y=x+5$, el valor de y será mayor que el valor de $x$. Subraya la respuesta correcta.

Siempre Nunca A veces

Justifica tu respuesta.

32. Si $y=7+x$, ¿qué les pasa a los valores de $y$ cuando los valores de $x$ aumentan?

33. Te encuentras en una papeleria en donde se hacen fotocopias. Para evitarse estar haciendo multiplicaciones el empleado tiene ta siguiente tabla:

\begin{tabular}{|c|c|}
\hline Nomero de copias & Precio \\
\hline 5 & $\$ 6,25$ \\
\hline 10 & $\$ 12,50$ \\
\hline 15 & \\
\hline 25 & $\$ 25,00$ \\
\hline 35 & $\$ 31,25$ \\
\hline 100 & \\
\hline
\end{tabular}

Completa la tabla 
34. Escribe la regla general si n denota el número de copias.

Observa la siguiente tabla y contesta las preguntas.

\begin{tabular}{|c|c|}
\hline Tiempo (seg.) & Velocidad (m/seg.) \\
\hline 0 & 0 \\
\hline 10 & 30 \\
\hline 15 & 60 \\
\hline 20 & \\
\hline 25 & \\
\hline 35 & \\
\hline 50 & \\
\hline 60 & \\
\hline
\end{tabular}

35. Completa la tabla

36. Si aumenta el tiempo, ¿qué le pasa a la velocidad, aumenta o disminuye?

37. En una hoja aparte, sobre un sistema de coordenadas marca los puntos de la tabla y únelos trazando aproximadamente una curva.

38. ¿Cuál es la vetocidad a los 15 segundos?

39. Fscribe la regla que asocia los números de la lista de la izquierda con los números de la lista de la derecha.

40. La variable dependiente en este ejemplo es

41. La variable independiente es

\section{Considera la siguiente expresión $y=3+x$.}

42. Si queremos que el valor de y sea 10 , ¿qué valor debe tener $x$ ?

43. Si queremos que el valor de $y$ sea 3 , ¿qué valor debe tener $x$ ?

44. Si queremos que los valores de $y$ sean mayores que 3 pero más pequeños que 10 , ¿qué valores puede tomar $x$ ? 45. Si $x$ toma valores entre 8 y 15 , ¿entre qué valores caerán los valores de y ?

46. De las siguientes dos expresiones $n+2$ y $2 x n$, ¿cuál es más grande? Justiftica tu respuesta 
Observa la siguiente tabia y contesta las preguntas.

\begin{tabular}{|c|c|}
\hline Hora & Imeca \\
\hline 0 & 50 \\
10 & 90 \\
15 & 180 \\
20 & 190 \\
22 & 180 \\
\hline
\end{tabular}

47. Si aumenta la hora, ¿qué le pasa al índice IMECA, aumenta o disminuye?

48. ¿Puedes predecir cuál es el vajor del índice IMECA a tas 18 horas ?

49. Escribe, de ser posible, la regla que asocia a la variable índice IMECA con la variable hora.

50. Traza, en la parte inferior de ta hoja, la siguiente gráfica:

\begin{tabular}{|c|c|}
\hline$x$ & $y$ \\
\hline 0 & 0 \\
10 & 100 \\
15 & \\
20 & 400 \\
25 & \\
-10 & 100 \\
-15 & 225 \\
-20 & \\
\hline
\end{tabular}

E1 peso de la mercancía que se compra en el mercado se mide con una báscula. En el puesto de Don Panchito, por cada kilogramo de peso la charola de la báscula se desplaza $4 \mathrm{~cm}$.

51. Encuentra una relación entre el peso de la compra y el desplazamiento de la charola.

52. Si la charola se desplaza $10,5 \mathrm{~cm}$ al pesar una bolsa de manzanas, ¿cuántos kilos pesa la bolsa? 
